\title{
Lack of 'acid reversal' of myofibrillar adenosine triphosphatase in masticatory muscle fibres of rhesus monkeys
}

\author{
LEOC. MAXWELL*, DAVID S. CARLSON and \\ CAROLE. BRANGWYN \\ Departments of Physiology and Anatomy, and The Center for Human Growth and Development, The \\ University of Michigan, Ann Arbor, Michigan 48109, U.S.A.
}

Received 8 November 1979

\begin{abstract}
Summary
Myofibrillar adenosine triphosphatase (ATPase) activity was demonstrated in sections of masseter and temporalis muscles and of selected limb muscles of adult rhesus monkeys. Incubations were performed either with no pre-treatment or after prior incubation in alkaline

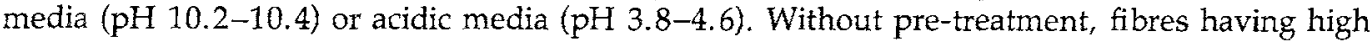
or low ATPase activity were observed in limb and masticatory muscles. Following alkaline pre-incubation the difference between high and low ATPase of limb muscle fibres is accentuated, whereas pre-incubation in acidic media $(\mathrm{pH} \mathrm{4.3)}$ results in inhibition of high and potentiation of low ATPase activities ('acid reversal'). While pre-incubation of masticatory muscle sections at $\mathrm{pH} 10.2$ accentuates differences in ATPase activity, pre-incubation at $\mathrm{pH} 10.4$ abolishes ATPase activity. In contrast, masticatory muscle fibres showed no reversal of ATPase activity following acidic pre-incubation ( $\mathrm{pH} \mathrm{4.3).} \mathrm{Pre-incubation} \mathrm{at} \mathrm{pH} 3.8$ abolished the ATPase activity of both limb and masticatory muscle fibres. The biochemical basis for the differences in ATPase histochemistry between masticatory and limb muscles is not known.
\end{abstract}

\section{Introduction}

Reversal of the myofibrillar ATPase reaction following pre-incubation of muscle cross-sections in acidic media was first reported by Drews \& Engel in 1966. They observed reversal when the pre-incubation medium contained EDTA at pH 4.0-4.7. However, assorted other media at $\mathrm{pH} 4.5$ failed to demonstrate the reversal effect. Nevertheless, the phenomenon has been attributed to differences in the $\mathrm{pH}$ lability of myofibrillar ATPase amongst fibre types, and was refined by Brooke \& Kaiser

\footnotetext{
*Present address: Department of Physiology, The University of Texas, Health Science Center at San Antonio, 7703 Floyd Curl Drive, San Antonio, Texas 78284, U.S.A. 
(1970a, b) to permit demonstration of several types of fibres based upon $\mathrm{pH}$ lability. They identified Type I fibres having low activity in the routine myofibrillar ATPase reaction at $\mathrm{pH} 9.4$ but with increased activity following $\mathrm{pH} 4.3$ pre-incubation, and Type II fibres with high activity in the routine $\mathrm{pH} 9.4$ assay which was inhibited by acidic pre-incubation. Type II fibres were further classified as Type II A if inhibition was complete with $\mathrm{pH} 4.5$ pre-incubation, Type II B if $\mathrm{pH} 4.3$ pre-incubation was required for complete inhibition and Type II C if inhibition was not complete unless pre-incubation $\mathrm{pH}$ was less than 4.0. These characteristics have been useful in classifying muscle fibres of limb skeletal muscles of humans (Meijer \& Vossenberg, 1977; Brooke \& Kaiser, 1970b; Askansas \& Engel, 1975) and laboratory animals (Samaha et al., 1970; Guth \& Samaha, 1972; Khan, 1977).

During the histochemical analysis of fibres of masseter and temporalis muscles of the rhesus monkey (Maxwell et al., 1979), we observed deviations from the $\mathrm{pH}$ lability characterisitcs of limb muscles. It is the purpose of this report to present these histochemical findings on masseter and temporalis muscles of rhesus monkeys and to compare them with observations on simultaneously incubated cross-sections of limb muscles from rhesus monkeys and other species.

\section{Methods}

Small cubes of muscle $\left(3-5 \mathrm{~mm}^{3}\right)$ were excised from anaesthetized animals. Samples were obtained from the masseter and temporalis muscles of 10 rhesus monkeys from standardized sites described previously (Maxwell et al., 1979a). Soleus and extensor digitorum longus muscles of monkeys were also sampled and processed simultaneously with the masticatory muscle samples. Soleus and extensor digitorum longus muscles of four rats and seven cats, and extensor digitorum longus muscles of six dogs were also studied. The cubes of muscle were mounted in a small amount of Cryoform and quick frozen in isopentane cooled with dry ice. Cross-sections, $14-20 \mu \mathrm{m}$ thick, were cut in a cryostat at $-20^{\circ} \mathrm{C}$, adhered to cover glasses, allowed to thaw and air-dried at room temperature for approximately $20 \mathrm{~min}$. Serial sections were then incubated for demonstration of succinic acid dehydrogenase (SDH) activity (Nachlas et al., 1957) or myofibrillar ATPase activity (Chayen et al., 1973) with or without prior incubation. The ATPase medium contained: $20 \mathrm{mM}$ sodium barbital, $100 \mathrm{mM} \mathrm{CaCl}$ and 1.9 mM ATP. The $\mathrm{pH}$ was adjusted to 9.4 at $37^{\circ} \mathrm{C}$. Incubation time was standardized to $20 \mathrm{~min}$ at $37^{\circ} \mathrm{C}$. Alkaline pre-incubation was performed in this medium without ATP and with $\mathrm{pH}$ adjusted to 10.2-10.4. Pre-incubation of limb muscle sections for $15 \mathrm{~min}$ at $\mathrm{pH} 10.4$ and $37^{\circ} \mathrm{C}$ in this medium yields greater contrast between Type I and Type II fibres. Masticatory muscles were pre-incubated at $\mathrm{pH} 10.2$ since higher $\mathrm{pH}$ often completely inhibited myofibrillar ATPase in all fibres. Acidic pre-incubations were for $5 \mathrm{~min}$ at room temperature in a medium containing: $24 \mathrm{~mm}$ sodium acetate, $14 \mathrm{~mm}$ sodium barbital and $\mathrm{HCl}$ to adjust $\mathrm{pH}$ to values of 3.8 to 4.6 . Whenever possible, masticatory muscle sections were pre-incubated and incubated in the same staining dish with sections of limb muscles. This ensured that differences observed were inherent in the muscles and not due to variations in media.

After incubations and colour development were completed, sections were dehydrated through a series of ethanols, cleared in xylene and mounted with Permount. Individual fibres were identified in each of the serial sections and a histochemical profile for each fibre determined. Photomicrographs were taken of representative muscle samples. Fibres of limb 
muscles were classified as Type I if the activity was low in the routine myofibrillar ATPase assay either with no pre-incubation or with alkaline pre-incubation, but was high following pre-incubation at $\mathrm{pH}$ 4.3; Type II $\mathrm{A}$ if activity in the routine assay was high, but was inhibited by pre-incubation at $\mathrm{pH} 4.5$; Type II $\mathrm{B}$ if pre-incubation at $\mathrm{pH} 4.3$ was required for complete inhibition; and as Type II C if $\mathrm{pH} 4.3$ pre-incubation did not completely inhibit the myofibrillar ATPase activity in a Type II fibre (Brooke \& Kaiser, 1970a, b). In limb muscles, most fibres were Types I, II A or II B and generally correspond to slow, fast-fatigue resistant and fast-fatiguable fibres classifed by Burke et al. (1971), although exceptions to this correlation were sometimes observed. An occasional Type II C fibre occurs. Since histochemical characteristics of muscle fibres of masticatory muscles prevented classification on the basis of $\mathrm{pH}$ lability, these fibres were classified only as Type I or Type II based on myofibrillar ATPase activity demonstrated with no pre-incubation or after alkaline pre-incubation at $\mathrm{pH} 10.2$. Subclassification of Type II fibres was based on SDH activity.

\section{Results}

Rhesus monkey limb muscle

A series of cross-sections of an extensor digitorum longus muscle of a rhesus monkey incubated for SDH activity and myofibrillar ATPase with alkaline pre-incubation at $\mathrm{pH} 10.4$ or acidic pre-incubation at $\mathrm{pH} 4.5,4.3$ or 3.8 (Fig. 1) demonstrate activity patterns illustrating the nomenclature of Brooke \& Kaiser (1970). Type I fibres have high .SDH activity, low myofibrillar ATPase after alkaline pre-incubation and high ATPase after acidic pre-incubation in the range of $\mathrm{pH}$ 4.6-4.3. Type II A fibres have high SDH activity and high myofibrillar ATPase which is inhibited by pre-incubation at a $\mathrm{pH}$ less than 4.5. Type II B fibres have low SDH activity and high myofibrillar ATPase which is inhibited by pre-incubation at $\mathrm{pH}$ less than 4.3. An occasionally observed Type II C fibre (not illustrated) has high SDH activity and demonstrates high myofibrillar ATPase which is not completely inhibited by pre-incubation at $\mathrm{pH} 4.3$ or 4.5 . Although most Type II fibres with high $\mathrm{SDH}$ activity were Type II A, exceptions were occasionally observed (for example, see the fibre denoted by the arrow in Fig. 1).

Dog limb muscle

Extensor digitorum longus muscle of dogs are mixed muscles comprised of Type I and Type II fibres (Fig. 2). All of the fibres in dog muscle have high SDH activity (Mlaxwell et al., 1977) and correspond histochemically to Type I and Type II A fibres. Reversal of the ATPase reaction is complete with pre-incubation at $\mathrm{pH} 4.5$. Pre-incubation at pH 3.8 completely inhibits myofibrillar ATPase.

\section{Rat limb muscle}

Extensor digitorum longus muscle of rats have predominantly three types of muscle fibres (Fig. 3) corresponding to Types I, II A and II B. Pre-incubation at $\mathrm{pH} 4.3$ is required to inhibit the myofibrillar ATPase in most Type II fibres. A Type II C fibre, illustrated in Fig. 3, has high myofibrillar ATPase both in the routine myofibrillar 


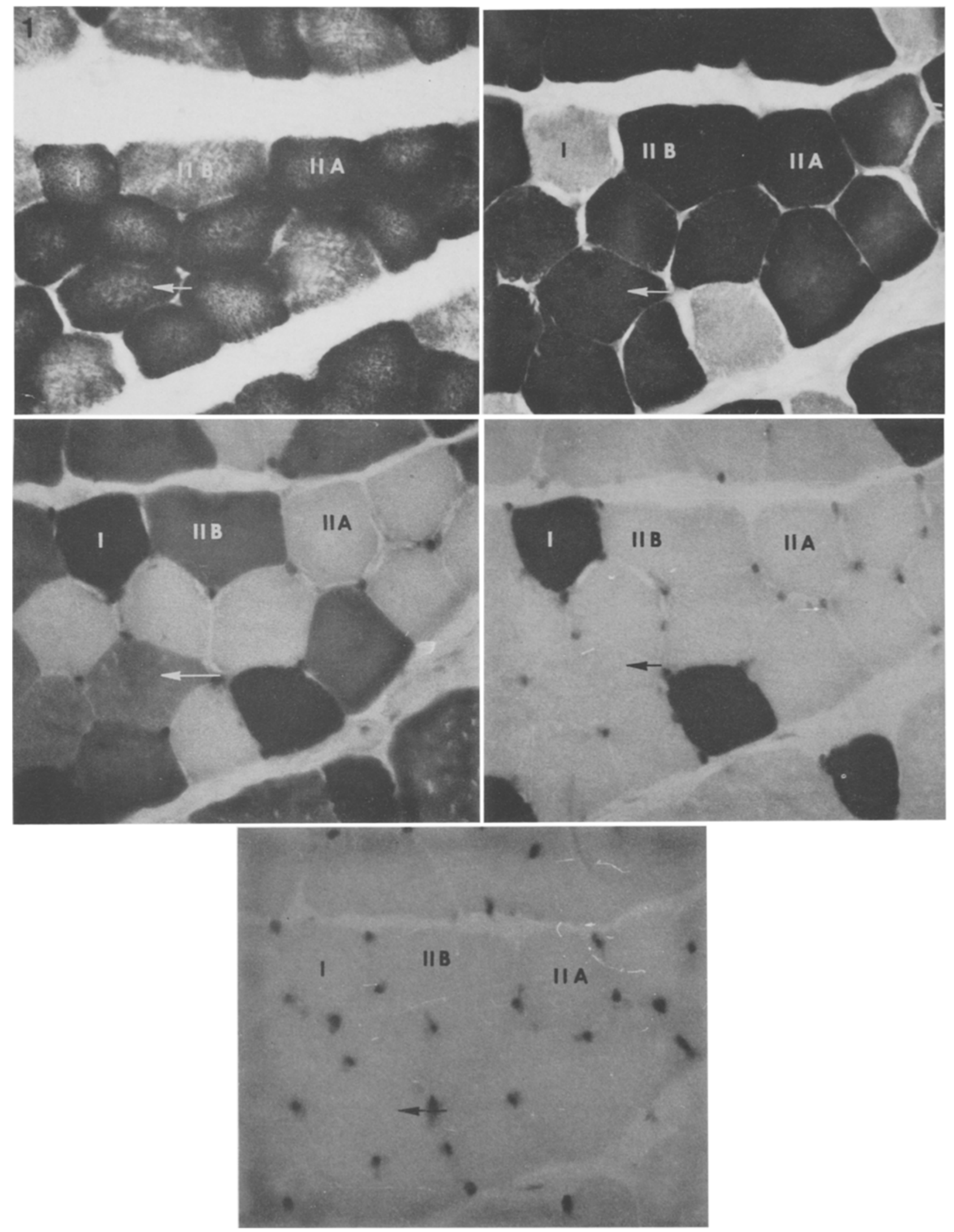



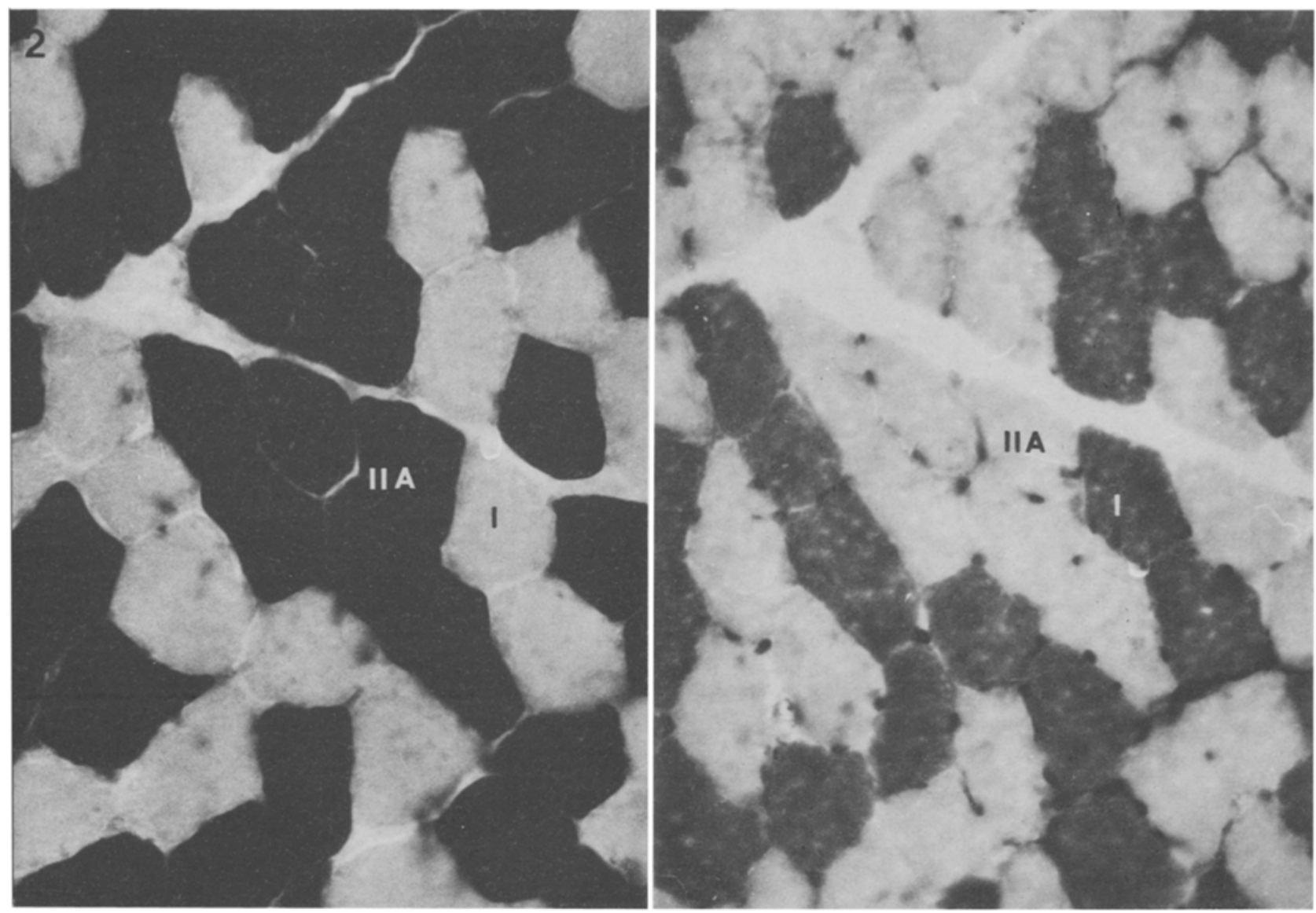

Fig. 2. Cross-sections of an extensor digitorum longus muscle of a dog were incubated for myofibrillar ATPase following alkaline pre-incubation at $\mathrm{pH} 10.4$ (left) and acidic pre-incubation at $\mathrm{pH} 4.3$ (right). Reversal of the ATPase pattern by acidic pre-incubation is demonstrated by all fibres. $\times 175$.

ATPase assay, and following pre-incubation at $\mathrm{pH} \mathrm{4.3.} \mathrm{This} \mathrm{fibre} \mathrm{also} \mathrm{has} \mathrm{high} \mathrm{SDH}$ activity and would be classified fast-fatigue resistant by the nomenclature of Burke $e t$ al. (1971).

Fig. 1. Cross-sections of an extensor digitorum longus muscle from a rhesus monkey were incubated for histochemical demonstration of succinic acid dehydrogenase (SDH) (top left), and myofibrillar ATPase following alkaline pre-incubation at $\mathrm{pH} 10.4$ (top right) or acidic pre-incubations at $\mathrm{pH} 4.5$ (middle left), 4.3 (middle right) or 3.8 (bottom). Type I, II A and II $B$ fibres are illustrated. Note that the myofibrillar ATPase activity of Type II B fibres is not completely inhibited by the pre-incubation at $\mathrm{pH}$ 4.5. A Type II B fibre with high SDH activity is marked by an arrow. Reversal of the ATPase pattern is demonstrated by all fibres. Muscle fibre ATPase activity is completely inhibited by pre-incubation at pH 3.8. $\times 175$. 

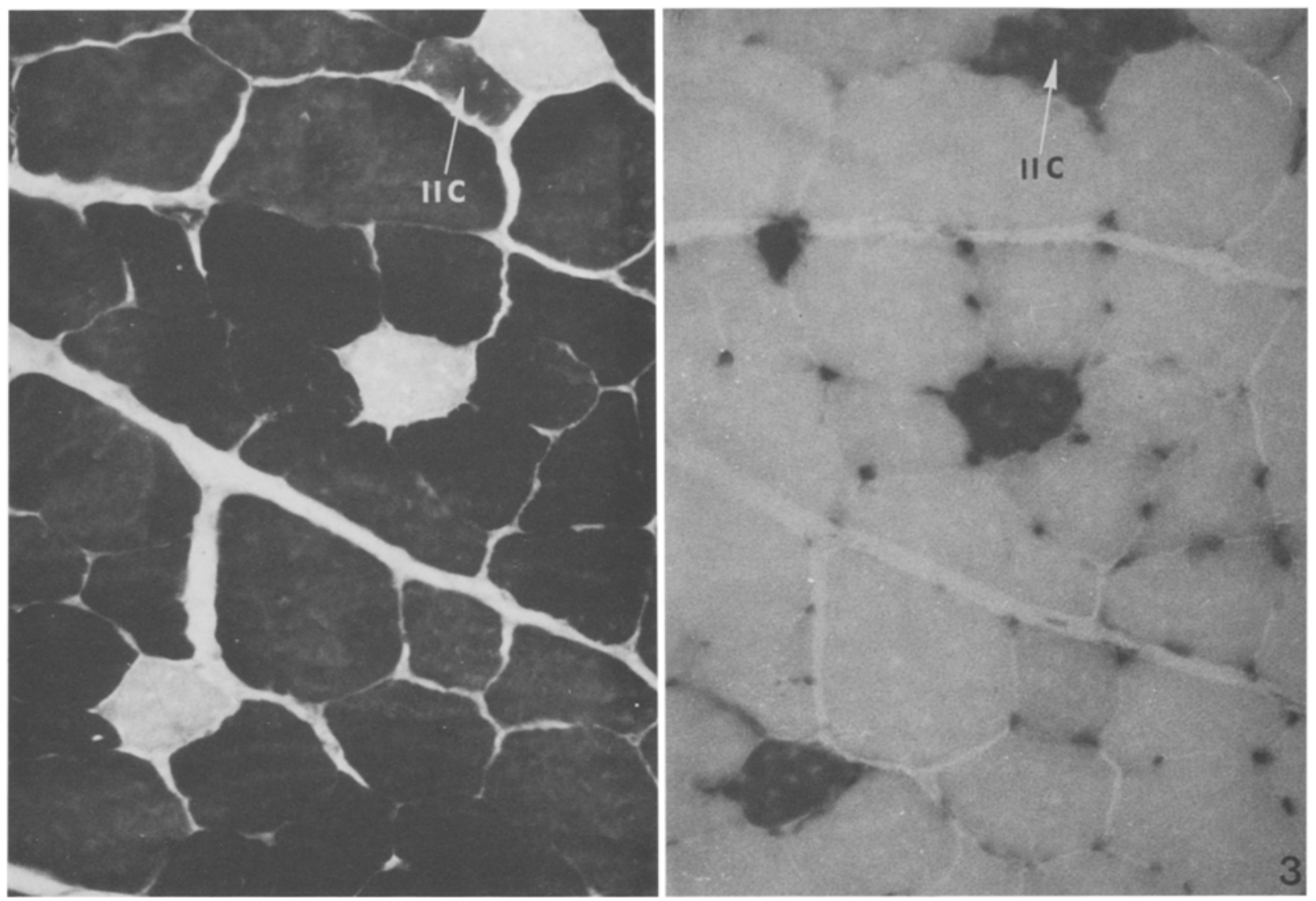

Fig. 3. Cross-sections of an extensor digitorum longus muscle of a rat were incubated for myofibrillar ATPase after alkaline pre-incubation at $\mathrm{pH} 10.4$ (left) and acidic pre-incubation at $\mathrm{pH}$ 4.3. Reversal of ATPase is shown by these fibres except for the Type II C fibre in which ATPase was not inhibited by pre-incubation at $\mathrm{pH} 4.3 . \times 175$.

Other limb muscles

Fibres of soleus muscles of monkeys, cats and rats, and extensor digitorum longus muscles of cats have characteristics similar to those described for limb muscles in Figs, 1-3.

Fig. 4. Cross-sections of a temporalis muscle from a rhesus monkey were incubated for myofibrillar ATPase without prior incubation (top left), after alkaline pre-incubation at $\mathrm{pH} 10.2$ (top right), and after acid pre-incubations at $\mathrm{pH} 4.5$ (bottom left) and 4.3 (bottom right). Note the intensification of contrast between Type I and Type II fibres following alkaline pre-incubation, but the lack of effect of acidic pre-incubations. $\times 175$. 


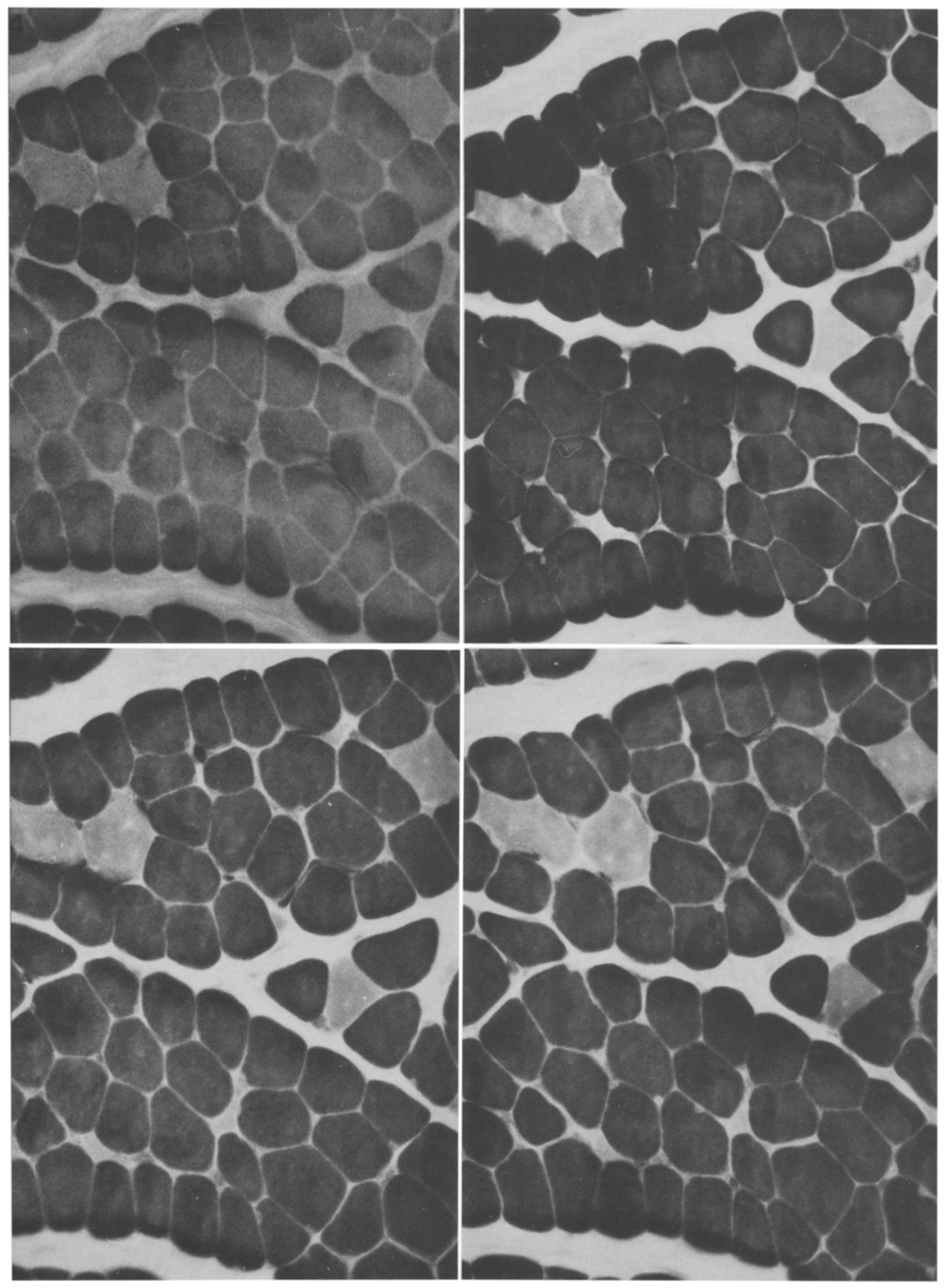


Rhesus monkey masticatory muscles

Deviations from the limb muscle fibre responses to pre-incubation were observed for masseter and temporalis muscles of rhesus monkeys. A series of cross-sections of temporalis muscle illustrate these results (Fig. 4). Fibres of masseter muscle behave similarly. The differences between masticatory muscle fibres and limb muscle fibres are: (1) in order to improve contrast between Type I and Type II fibres, we have held the $\mathrm{pH}$ of the alkaline pre-incubation medium for fibres of masticatory muscles to 10.2 in order to avoid complete inhibition of myofibrillar ATPase, whereas pre-incubation $\mathrm{pH}$ as high as 10.4 does not interfere with expression of activity by Type II fibres of limb muscles; (2) pre-incubation of masticatory muscles sections at $\mathrm{pH}$ less than 4.6 produces neither the inhibition of Type II nor the activation of Type I fibre activity normally seen in limb muscle sections. Thus, the acid reversal phenomenon was not demonstrated, and we have not been able to classify fibres into subtypes based on the reaction following acid pre-incubation. It is emphasized that masticatory muscle sections were subjected to the same media, often in the same dish, and at the same time as limb muscle sections were processed.

\section{Discussion}

Widespread use of histochemical technique for the characterization of muscle fibres has led to a proliferation of nomenclature systems. Most of these identify three main types of muscle fibres, with differences in histochemical profiles and physiological properties.

Two types of classification systems predominate. In the first, fibres are classified on the basis of the activities of an oxidative enzyme, such as SDH, and of myofibrillar ATPase assayed at $\mathrm{pH} 9.4$ with no pre-incubation or following an alkaline pre-incubation. The three types of fibres so identified have been variously called: intermediate, red and white (Edgerton \& Simpson, 1969); slow-twitch oxidative, fast-twitch oxidative-glycolytic and fast-twitch glycolytic (Peter et al., 1972); high oxidative-slow, high oxidative-fast and low oxidative-fast (Maxwell et al., 1973); $\beta$ red, $\alpha$ red and $\alpha$ white (Ashmore \& Doerr, 1971) or slow twitch, fast twitch-fatigue resistant and fast twitch-fatiguable (Burke et al., 1971), respectively. Although the nomenclatures differ, fibre characteristics are similar amongst these schemes. The nomenclature of Burke et al. (1971) is based upon the correlation between physiological recording of contractile properties and the histochemical characteristics of single motor units of gastrocnemius muscle of cats.

In the second system, myofibrillar ATPase activity demonstrated at $\mathrm{pH} 9.4$ with no pre-incubation or following an alkaline pre-incubation is used to classify limb muscle fibres as Type I (light) or Type II (dark). Type II fibres are then subtyped based upon differences in lability following acidic pre-incubation. Type I, Type II A and Type II B fibres are usually thus identified (Brooke \& Kaiser, 1970a, b). Since Type II A fibres have higher oxidative enzyme activity than Type II B fibres (Brooke \& Kaiser, 
$1970 a, b)$, these types of fibres usually correlate well with the three types of fibres classified on the basis of oxidative enzyme and myofibrillar ATPase activities. The results of the current study support this generalization for most fibres of limb skeletal muscles of monkeys, cats and dogs, but occasional Type II B fibres with high SDH activity were observed. Type II B fibres with high SDH activity have also been observed by Khan (1977). However, the good general correlation between these two types of nomenclature and classification systems for $\operatorname{limb}$ muscle fibres permits comparison of results from different investigators and lends further support to the concept that three major types of limb skeletal muscle fibres exist.

The fibres of the masticatory muscles, masseter and temporalis of rhesus monkeys do not fit the patterns observed for limb muscle fibres. Compared to myofibrillar ATPase performed without prior incubation, the treatment of limb muscle cross-sections at $\mathrm{pH} 10.4$ in the presence of $\mathrm{Ca}^{2+}$ accentuates differences amongst fibres. However, pre-incubation of masticatory muscle sections at $\mathrm{pH} 10.4$ often abolishes myofibrillar ATPase activity in all fibres. Without a non pre-incubated section as control, conditions which work well for limb muscles could yield erroneous interpretations for sections of masticatory muscle. We have determined experimentally that $\mathrm{pH} 10.2$ is usually safe for pre-incubation of sections of masseter and temporalis muscles. These differences between $\operatorname{limb}$ and masticatory muscles of rhesus monkeys likely indicate that these alkaline $\mathrm{pH}$ values are close to the maximum $\mathrm{pH}$ which myofibrillar ATPase can tolerate.

Differences between limb and masticatory muscle fibres in acid lability are more pronounced. Following pre-incubation in the $\mathrm{pH}$ range from 4.3 to 4.6 , the myofibrillar ATPase of Type I fibres of limb muscles is augmented but that of masticatory muscle Type I fibres is unchanged relative to non pre-incubated myofibrillar ATPase sections. Using this same range of pre-incubation $\mathrm{pH}$, the myofibrillar ATPase activity of Type II fibres of limb muscles is inhibited, with the more oxidative fibres being most easily inhibited, whereas the myofibrillar ATPase of Type II fibres of masticatory muscles is unchanged relative to a non pre-incubated section. Thus, under conditions where 'acid reversal' of myofibrillar ATPase is readily demonstrated in limb muscle fibres, we have failed to demonstrate the phenomenon in rhesus monkey masseter or temporalis muscle sections. Classification of masticatory muscle fibres of rhesus monkeys relies on demonstration of SDH and myofibrillar ATPase either without pre-incubation or following alkaline pre-incubation (Maxwell et al., 1979a, b), and classification by the system of Brooke \& Kaiser $(1970 a, b)$ is not possible. Failure to show 'acid reversal' has also been reported in muscles of humans afflicted with neuromuscular disease (Meijer \& Vossenberg, 1977), in masticatory muscle fibres of foetal humans (Ringqvist et al., 1977) and in some Type II fibres in masticatory muscles of adult humans (Ringqvist, 1973).

Fibres which would be classified Type II based on high ATPase activity at pH 9.4 without pre-incubation, but whose ATPase activity is not inhibited by acid 
pre-incubation have been reported for immature, developing muscles (Guth \& Samaha, 1972). Such fibres are also infrequently seen in adult muscles and have been classified Type II C by Brooke \& Kaiser $(1970 a, b)$. The histochemical behaviour of Type II fibres in rhesus monkey masticatory muscles is consistent with the pattern for II C fibres. However, since the muscles were taken from adult animals, and few immature fibres were observed in limb muscles from these animals, a high proportion of immature fibres seems unlikely. Furthermore, the lack of 'acid reversal' by Type I fibres of rhesus monkey masticatory muscles is not satisfactorily explained by an immature fibre hypothesis. We conclude, therefore, that the fibres of masticatory muscles of adult rhesus monkeys are likely to be mature, but that for unknown physical or chemical reasons they fail to demonstrate myofibrillar ATPase reactions consistent with fibre types of limb muscle fibres.

The correlation between various classification systems and the correlation between acid lability and oxidative capacity of Type II fibres for limb muscles could lull investigators into attempting to classify muscle fibres based on very few enzymatic assays. For example, three types of fibres can be classified in limb muscles of rhesus monkeys using myofibrillar ATPase after pre-incubation at $\mathrm{pH} 4.5$. These results would correlate quite well with classification based upon SDH and myofibrillar ATPase activities. However, this approach would have been very misleading for the masseter and temporalis muscles of rhesus monkeys; and there are probably other muscles in a variety of species which may not behave histochemically as would be predicted based on the results of limb muscle research. Both muscle and species specificity may be important. The safe approach, particularly with a muscle of unknown characteristics, is still a battery of histochemical reactions including an oxidative enzyme and myofibrillar ATPase without pre-incubation as fundamental assays, with myofibrillar ATPase following various pre-incubations, and other enzymatic assays added as secondary support for classification or to answer specific histochemical questions.

\section{Acknowledgements}

Technical assistance was provided by Michael Kielb.

This research was supported by NIH Grants DE 04227 and DE 05232 and Contract DE 52478.

\section{References}

ASHMORE, C. R. \& DOERR, L. (1971) Comparative aspects of muscle fiber types in different species. Expl Neurol. 31, 408-18.

ASKANSAS, V. \& ENGEL, W. K. (1975) Distinct subtypes of Type I fibers in human skeletal muscle. Neurology 25, 879-87.

BROOKE, M. H. \& KAISER, K. K. (1970a) Muscle fiber types: how many and what kind. Archs Neurol. 23, 369-79. 
BROOKE, M. H. \& KAISER, K. K. (1970b) Three 'myosin adenosine triphosphatase' systems: the nature of their $\mathrm{pH}$ lability and sulfhydryl dependence. J. Histochem. Cytochem. 18, $670-2$.

BURKE, R. E., LEVINE, P. N., ZAJAC, F. E. III, TSAIRIS, P. \& ENGEL, W. K. (1971) Mammalian motor units: physiological correlates of three types in cat gastrocnemius. Science, N.Y. 174, 709-12.

CHAYen, J., BItensky, L. \& BUtCher, R. G. (1973) Practical Histochemistry. pp. 122-124. New York: Wiley.

DREWS, G. A. \& ENGEL, W. K. (1966) Reversal of the ATPase reaction in muscle fibers by EDTA. Nature 212, 1551-3.

EDGERTON, V. R. \& SIMPSON, D. R. (1969) The intermediate muscle fiber of rats and guinea pigs. J. Histochem. Cytochem. 17, 828-38.

GUTH, L. \& SAMAHA, F. J. (1972) Erroneous interpretations which may result from application of the 'myofibrillar ATPase' histochemical procedure to developing muscle. Expl Neurol. 34, 465-75.

KHAN, M. A. (1977) The histoenzymology of striated muscle fibers: an overview. Cell. molec. Biol. 22, 383-93.

MAXWELL, L. C., BARCLAY, J. K., MOHRMAN, D. E. \& FAULKNER, J. A. (1977) Physiological characteristics of skeletal muscles of dogs and cats. Am. J. Physiol., Cell Physiol. 2, C14-18.

MAXWELl, L. C., CARLSON, D. S., McNAMARA, J. A. JR. \& FAUlKNeR, J. A. (1979a) Histochemical characteristics of the masseter and temporalis muscles of the rhesus monkey (Macaca mulatta). Anat. Rec. 193, 389-401.

MAXWELl, L. C., McNAMARA, J. A. JR., CARLsON, D. S. \& FAulKNeR, J. A. (1979b) Histochemical characteristics of fibers of masseter and temporalis muscles of edentulous rhesus monkeys (Macaca mulatta). Arch. oral Biol. (in press).

MAXWELL, L. C., FAULKNER, J. A. \& LIEBERMAN, D. A. (1973) Histochemical manifestations of age and endurance training in skeletal muscle fibers. Am. J. Physiol. 344, 356-61.

MEIJER, A. E. F. H. \& VOSSENBERG, R. P. M. (1977) The value of enzyme histochemical techniques in the classification of fiber types of human skeletal muscle. Histochemistry $\mathbf{5 2}$, $45-53$.

NACHLAS, M., TSOU, M. K., de SOUZE, E., CHENG, C. \& SELIGMAN, A. M. (1957) Cytochemical demonstration of succinic dehydrogenase by the use of a new $p$-nitrophenyl substituted ditetrazole. J. Histochem. Cytochem. 5, 420-36.

PETER, J. B., BARNARD, R. J., EDGERTON, V. R., GILLESPIE, C. A. \& STEMPEL, K. A. (1972) Metabolic profiles of three fiber types of skeletal muscles in guinea pigs and rabbits. Biochemistry 11, 2627-33.

RINGQVIST, M. (1973) Histochemical enzyme profiles of fibers in human masseter muscles with special regard to fibers with intermediate myofibrillar ATPase reaction. J. Neurol. Sci. $18,133-41$.

RINGQVIST, M., RINGQVIST, I. \& THORNELL, L. A. (1977) Differentiation of fibers in human masseter, temporalis and biceps brachii muscles. J. Neurol. Sci. 32, 265-73.

SAMAHA, F. J., GUTH, L. \& ALBERS, R. W. (1970) Phenotypic differences between the actomyosin ATPase of the three fiber types of mammalian skeletal muscle. Expl Neurol. $26,120-5$. 